\title{
Derecho Aéreo
}

\section{NOTAS CONCERNIENTES AL REGIMEN INTERNACIONAL DE RESPONSABILIDAD POR LOS DANOS CAUSADOS POR VEHICULOS ESPACIALES}

por RENE H. MANKIEWICS

(Consejero Juridico de la O. A. C. I.) (1)

Traducción y comentario de Jaime Cruzat Corvera, ayudante ce Dere. cho Aereo del Seminario de Derecho Público.

\section{Consideraciones preliminares}

1. - Esta nota trata esencialmente de la responsabilidad por los daños causados por un vehículo espacial diferente del país del cual fue lanzado. En efecto, las disposiciones de las leyes nacionales sobre la responsabilidad cuasidelictual aplicables al Iugar donde se produce el el daño aseguran desde ya la indemnización justa de la víctima, sea cual fuere el lugar o la actividad donde el daño fue producido. Sin embargo, la ausencia de una relación directa de causa a efecto puede dificultar la acción de la víctima. A título de ejemplo podemos imaginar el caso de un vehículo caido en una represa, lo que produce la inundación de los terrenos de cultivo. El daño directo es causado a la represa, la inundación de los campos no es más que un daño indirecto. ¿Puede el agricultor reclamar daños y perjuicios al đueño del vehículo? Las leyes nacionales sobre esto no son uniformes.

Recordemos por otra parte, que las aplicaciones y ámbito de las reglas nacionales sobre la responsabilidad cuasidelictual se determinan según el derecho Internacional Privado cuando el daño

11) Traducido de la Revista Francesa de Derecho Aéreo de Enero-Marzo 1963. se produjo fuera del Estado en el cual el daño fue efectivamente causado. Además, puede haber una laguna legal o vacuum juridique cuando el hecho cuyo resultado es el daño tuvo lugar en el espacio extraterrestre que escapa a toda soberanía.

2. Es importante elaborar un régimen internacional de responsabilidad por los daños causados por los vehiculos espaciales, y cllo en el interés, a la v'ez, de la seguridad y uniformidad del derecho. Se puede esperar que estas reglas uniformes internacionales de derecho espacial se impondrán también para la reglamentación de la responsabilidad en el derecho nacional, asegurando así la uniformidad de los derechos internacionales y nacionales.

3. Antes de abordar los principios que deberán formar la base de éste de recho es útil dar algunas nociones preliminares:

$1^{9}$ El presente trabajo no tratará de la responeabilidad por los daños causados por un vehículo espacial en el interior del país de lanzamiento, aunque estos daños sean causados a los nacionales $o$ extranjeros. Este problema es, 
en efecto. del resorte del derecho nacional, mientras que nuestro proposito es examinar las reglas susceptibles de gobernar la responsabilidad en el derecho internacional y de determinar las bases de un derecho uniforme que, a ejemplo del derecho aéreo privado internaciona! uniforme, evitaria el hecho de recurrir al siempre molesto arreglo de conflictos de leyes, y podría servir de modelo al derecho interno de los países que no han adoptado su derecho privado nacional a las nuevas condiciones de la era espacial.

$2^{\circ}$ Se supone que, tal como los aviolies, los vehículos espaciales llevarán marcas que permitirán identificar el Estado que los lanzó.

3o Nosotros entendemos por vehículo espacial toda máquina destinada a ir más allá del espacio aeronáutico. Esta máquina será, entonces, un vehículo espacial no solamente en el curso de sus evoluciones en el espacio extraeronáutico sino también antes de su lanzamiento, durante Ia travesía del espacio aeronáutico y después de su vuelta a la tie. rra. En efecto, será calificado como vehiculo espacial desde el momento en que esté completamente armadio hasta que se vuelva a desarmar.

Resulta de esta definición que el régimen de responsabilidad - como nosotros lo esperamos- de la navegación espacial será un derecho uniforme en el sentido de que una sola reglamentación se aplicará, sin importar el lugar geográfico en que el vehículo se encuentre al causar el daño.

Notamos, sin embargo, que el riesgo propio de las actividades espaciales no comienza más que en el momento de la puesta en marcha del vehículo, por to cual el régimen do responsabilidad que tratamos aquí no debe aplicarse sino a partir de éste momento.

Por otra parte suponiendo que su diseño técnico permitiera considerar el vehículo espacial como una aeronave durante su travesía en el espacio aeronáutico (a la ida y regreso) no se le puede someter durante éste lapso a las reglas del derecho aéreo.
En efecto, la naturaleza peculiar d: éste vehículo y las actividades a las cuales es destinado exigen la claboración de un régimen especial de responsabilidad, que se aplicaría de una manera continua desde el lanzamiento del aparato hasta el final de su existencia como vehículo espacial.

\section{GENEROS Y EXTENSION DE LOS DA- ÑOS SUSCEPTIBLES DE SER CAUSA- DOS POR VEHICULOS ESPACIALES}

4. Estos dan̆os son de dos clases. Por una parte, los daños resultantes del impacto (choque material) del vehículo mismo debido a las peripecias de sus evoluciones. Así, será imputable por ejemplo, a las evolucioncs del vehículo la caída de un avión a continuación de una maniobra de evasión, a fin de evitar una colisión con el vehículo.

Por otra parte, los daños pueden ser provocados por las actividades realizadas al partir el vehículo, a causa de las emisiones terrestres necesarias para su manejo. Ejemplos: daños resultantes de la interrupción de las comunicaciones terrestres o aéreas durante las emisiones radiales en la procedencia o ei destino del vehículo; daños causados por falsos mensajes de parte del vehículo, por alteraciones del clima y las condiciones meteorológicas imputables al funcionamiento del mismo

En efecto, como lo ha hecho notar recientemente el Secretario de Estado de los Estados Unidos de América, "el hombre es capaz de utilizar el espacio extraaeronáutico de manera de crear un peligro demasiado real para la civilización y para la vida misma sobre el globo terrestre".

6. No es necesario, pues, engañarse sobre la extensión y la importancia del riesgo que significa la utilización del espacio extraeronáutico para fines pacíficos. En efecto, en los momentos de la guerra fría, aún las actividades espaciales no militares, "pacíficas" en su sentido estricto, pueden desorganizar la vida económica de una nación, comprometer su seguridad, pueden alcanzar su orden interior y danar la salud de los 
hombres. Notemos así que Ios resultados de una explosión nuclear con miras científicas son susceptibles de producir consecuencias biológicas para las generaciones presentes y futuras. Las víctimas de estas actividades pueden ser tanto la colectividad como los individuos.

Los daños causados de esta manera pueden alcanzar proporciones fuera de los límites comunes.

7. Las reglas de responsabilidad internacional deben ser formuladas teniendo en cuenta estos elementos, en particular la enormidad de los daños que pueden resultar de las actividades espaciales. También debe contemplarse la indemnización al propietario de la casa que ha sido destruida por el impacto de un vehículo o de un objeto que ha sido lanzado, o de reglamentar la reparación del daño causado a un terreno agrícola que ha servido como campo de aterrizaje. Es preciso tener en consideración los daños inmensos que puede sufrir una región o un pais como consocuencia de una inundación o de un pánico provocado, sea por las actividades de un vehiculo espacial pacífico, sea por las emisiones de radio provenientes de tal vehículo.

8. Estos daños no son necesariamente atribuibles a la voluntad de perjudicar respecto de aquelios que controlan las evoluciones $y$ actividades del vehículo, pues pueden resultar ya sea de un mal funcionamiento de los telecomandos o de los aparatos de abordo, 0 , todavía, de una falsa maniobra del astronauta o del hecho de que éste se ha desvanecido y ha perdido el control de sus instrumentos.

9. Todos estos daños, el lanzamiento y las actividades de vehículos espaciales, presentan fenómenos sin precedentes. La reglamentación de la responsabilidad de los propietarios de los vehículos espaciales no se puede tener en cuenta.

Bien puede que ella repose sobre una apreciación exacta de estas nociones dc hecho, que sustraen los vehículos espaciales al derecho común de la responsabilidad y piden la elaboración de reglas jurídicas exorbitantes al derecho tradi- cional: "a hecho nuevo, derecho nuevo"; no serán, pues, válidos sino en la medida en que las reglas de responsabilidad sean adoptadas a las realidades técnicas y sociales de la era espacial.

Partiendo de estas reflexiones nos proponemos determinar las personas o entidades responsables de la reparación de daños causados por un vehículo espacial y sus actividades, y de buscar el fundamento y la extensión de su responsabilidad.

10. Un fenómeno también tan revolucionario como la explotación del es* pacio aeronáutico, a saber, la utilización de la energía atómica, ha conducido, con justa razón, a muchos Estados a abandonar, en materia de responsabilicad nuclear, las reglas tradicionales y a concluir un acuerdo que signifique un ruevo desarrollo del derecho de la responsabilidad. Esta actitud, más que las normas antiguas, debe también orientarse a la elaboración del derecho de la lesponsabilidad espacial.

\section{CONSIDERACIONES SOBRE LAS PER- SONAS Y ENTIDADES RESPONSA- BLES DE LOS DAÑOS}

11. En principio, la causa de un daño resultante de una actividad espacial puede ser imputada a diversos autores cue serán eventualmente responsables como deudores solidarios. A título de ejemplo, las hipótesis siguıentes pueden ser consideradas.

El daño puede resultar de un defecto $c$ de una mala preparación en el lanzamiento. Ejemplos: colisión con ntra máquina espacial o una aeronave, derivada del hecho de que la trayectoria y órbita han sido mal elegidas o mal calculadas, 0 , también del hecho de que las estaciones terrestres de control, por razones técnicas, no han podido asegurar que la máquina seguirá la trayectoria o la órbita predetorminada. La responsabilidad deberá, entonces, incumbir a aquellos que han organizado el lanzamiento o dispuesto las estaciones de control.

Por otra parte, cl accidente - colision o impacto de la máquina con un sitio no previsto- puede resultar del mal funcionamiento de un aparato instrumental 
de abordo. El responsable de este estrago será, según el caso, el "dispatcher" o el fabricante de los aparatos defectuosos.

En fin, el daño puede ser causado por fenómenos cósmicos o por un error de maniobra del astronauta. ¿Será necesario admitir en el primer caso que la intervención de una fuerza mayor suprime toda responsabilidad, y que el astronauta será personalmente responsable, en el segundo caso?

12. De todas maneras la victima tendrá grandes dificultades para establecer la causa precisa del hecho dañoso y de determinar la persona o el organismo responsable. Por otra parte, contados serán los casos en que la causa del daño quedará siempre ignorada.

Tal es todavía actualmente la situación en el campo de la aviación. Este es el porqué fueron llevadas a cabo las Convenciones de Varsovia y de Roma; desde entonces ocurre que, en el derecho común, la víctima no puede normalmente obtener indemnización sino que en la medida en que ella pueda probar el elemento causal de su parte.

13. Las consideraciones bosquejadas ilustran suficientemente la necesidad de elaborar un régimen de responsabilidad enteramente nuevo por los daños resultantes de las actividades espaciales. Por eso, puesto que la regla de derecho expresa el equilibrio entre los intereses antagónicos, el nacimiento de hechos $\mathrm{y}$, por consiguiente, de nuevos y profundos intereses, pide la puesta en práctica de un régimen que sea también original.

El método valedero consiste en tener en cuenta los problemas técnicos y evasuar los intereses económicos, sociales y políticos en juego en esta nueva aventura de la Humanidad.

\section{RESPONSABILIDAD EXCLUSIVA DEL ESTADO EN EL LANZAMIENTO DE UN VEHICULO ESPACIAL. FUNDA- MENTO Y LIMITES}

14. Todo vehículo espacial "pacifico" es virtualmente una máquina militar y nociva. Por eso, considerando los peli- gros para la humanidad, que son inherentes a toda actividad espacial, nosotros sugerimos mantener restringidos el lanzamiento y la explotación de los vehículos espaciales y reservarlos solamente a los Estados. La libertad de comercio y de industria debe ceder ante el interés general internacional. Tanto más cuanto que sólo un Estado puede garantizar eficazmente, subre el plano internacional, la inocuidad del vehículo y de sus actividades e impedir la utilización abusiva o nociva del espacio extraeronáutico.

Además, dada la naturaleza doble de todo vehículo espacial; debe sufrir una inspección previa al lanzamiento, mientras que este control no sea confiado a una organización internacional -que será, por muchas razones, la mejor solución- no podrá ser efectuado con las garantías necesarias sino que por el Estado que lo lanza .

15. Es preciso recordar que toda actividad espacial corre el riesgo de crear un monopolio de hecho, sea por el acaparamiento de la mejor órbita -0 de la más económica - sea a causa de la naturaleza misma de estas actividades. Pelo la monopolización de órbitas o de ventajas resultantes de la explotación del espacio extraeronáutico, parte del Universo y del patrimonic humano, es intolerable. Es preciso, entonces, que la utilización de este espacio sea el privilegio de los Estados, únicos capaces de asegurar, en el plano internacional, la participación de terceros en los beneficios que puedan derivarse.

Notemos, sin embargo, que este régimen de privilegio no impedirá a un Estado autorizar a una empresa privada dependiente de su autoridad para dedicarse a actividades espaciales, estando fntendido que el Estado mantendrá el control absoluto de sus actividades y responderá frente a tercaros. En fin, algunas convenciones internacionales y muchas legislaciones nacionales preverán, desde luego, que un Estado asegurará el lanzamiento de una máquina fabricada por un extraño del mismo modo que por una empresa privada. Estos casos están 
llamacios a multiplicarse en breve plazo. Ahora bien, "los dueños del infinito estarán en condiciones de controlar los climas sobre la tierra, de provocar la sequía o la inundación, de modificar los niveles del mar, de desviar la corriente del Golfo y de transformar un clima temperado en un clima frio". Así lo ha declarado el Vicepresidente de los Estados Unidos de América en su discurso ante las Naciones Unidas. Todo envío de un vehículo espacial es, entonces, asunto $\tan$ grave que debe evitarse toda repartición de responsabilidad. Las conclusiones siguientes parecen ineludibles.

16. Desde el momento que un vehículo puede causar grandes daños y molestar las actividades legítimas de otros vehiculos espaciales, parece inadmisible que la responsabilidad quede a cargo de instituciones privadas, hasta de sociedades comerciales poderosas. DeI mismo modo, el hecho de que cl vehículo haya estado entregado en préstamo por otro Estado distinto, no disminuirá la responsabilidad del Estaco que ha autorizado la partida hacia el espacio extraeronáutico. Resultaría así que no sería tolerable que un Estado acepte o autorice el lanzamiento de un vehiculo, sin ser informado por sus propios servicios administrativos acerca del carácter inofensivo de la máquina y de sus actividades, así como de los aparatos y procedimientos que aseguren el control y manejo a distancia.

17. Deberá, asimismo, preguntarse si el Estado en que se hace el lanzamiento no estaría obligado a asegurarse de antemano respecto del buen funcionamiento de las estaciones terrestres extranjeras cuya intervención será requerida para el control del vuelo y las actividades del vehiculo.

Sin embargo, en el plano del régimen que nosotros proponemos bastará que este Estado garantice la inocuidad del vehículo y de sus actividades y, desde ya, la eficacia y buen funcionamiento de los aparatos embarcados. Si el daño es causado por error o mal funcionamiento de una estación de control y de guía extran- jera, la responsabilidad por los daños que resulten incumbirá, en primer lugar, al Estado donde está situada esta estación. Corresponderá, sin embargo, al Estado que hace el lanzamiento tomar las medidas apropiadas para que estas estaciones recojan en tiempo útil las indicaciones necesarias para llevar a cabo sus funciones, $y$ asegurar por adelantado que ellas estén en situación de asumir el papel que les está asignado.

\section{RESPONSABILIDAD OBJETIVA E ILIMITADA}

18. En el estado actual de nuestros conocimientos científicos y técnicos, sólo la responsabilidad objetiva e ilimitada parece ofrecer una solución satisfactoria, acorde a la vez con la cvolución moderna de principios de responsabilidad y la evaluación justa de intereses en juego. Las razones de esta proposición, expuesta, por otra parte, con más detalles, pueden resumirse como sigue:

A pesar de un conocimiento más perfecto de hechos cósmicos y no obstante el dominio grandioso de la técnica del control de vehículos y de sus actividades, todo lanzamiento de un vehículo, toda actividad espacial, es una empresa que comparte, por su naturaleza, riesgos extremadamente graves. Aun cuando se hayan tomado todas las medidas y precauciones humanamente posibles según el estado actual de conocimientos científicos más precisos, el vehículo espacial puede causar daños $\mathrm{y}$, lo que es más, la causa directa de los daños puede quedar para siempre ignorada. En todo caso, el hecho perjudicial será siempre imprevisible o constituirá un caso de fuerza mayor. En fin, por largo tiempo todavía, toda actividad espacial será una experiencia original $\mathrm{y}$, por consiguiente, susceptible de engendrar perturbaciones insospechadas en el régimen físico del espacio, que serán afectados por su repercusión en la atmósfera o sobre la tierra. Es preciso, entonces, convenir en que la responsabilidad por un daño causado por una máquina espacial o con motivo de una actividad espacial debe ser objetiva, es decir, que efla es produ- 
cida por el hecho mismo del daño, en ausencia de toda culpa, y lo mismo en caso de fuerza mayor.

19. La adopción de este régimen encuadra con la evolución reciente del derecho de la responsabilidad. En la sociedad moderna, que dispone de sistemas de seguridad más completos, no se trata, pues, para garantizar la reparación de un daño, de atribuir la responsabilidad según la gravedad de la culpa, sobre la base de consideraciones de orden social. Consideraciones de política social exigen que la víctima pucda siempre obtener indemnización, cualquiera que saa el grado de culpa de una u otra parte.

Sea quien fuere el que deberá reparar el daño, corresponderá al autor tomar las medidas de seguridad que juzgue nocesarias.

En efecto, la previsión del daño y la posibilidad de evitarlo por las medidas apropiadas, no pueden, como otras veces, servir de base al régimen de responsabilidad en una sociedad técnica cuando, por una partc, la previsión ha llegado a ser cosa imposible o cuando, por la otra, puede ser siempre demostrado que la aplicación de técnicas más perfectas y una previsión más minuciosa y más clara podrá evitar el daño.

La responsabilidad objetiva por los daños causados por una máquina espacial o con ocasión de una actividad espacial corresponde a estos principios del derecho moderno.

Ellos han sido reconocidos en el plano internacional por la Convención de Roma (1952) sobre los daños causados en la superficie por aeronaves extranjeras $y$, recientemente, por las Convenciones sobre responsabilidad por los accidentes nucleares.

20. Este sistema encuentra, sin embargo, dificultades en el caso particula: cuando el daño es causado por muchos vehículos espaciales o por el choque entre diferentes actividades espaciales.

Ningún problema se produce, es verdad, para las víctimas, quienes, a su elección, se volverán contra uno u otro Estado responsables, o los perseguirán a todos a la vez. No es lo mismo en aquallo que concierne al caso más complejo de relaciones entre esos Estados.

Se han preconizado muchas soluciones para una situación algo análoga, a saber, en caso de choque entre aeronaves. para lo cual el proyecto de Convención elaborado por el Comité Jurídico de la O. A. C. I. previó igualmente un régimen de responsabilidad objetiva, pero limitaao. En virtud de este proyecto de Convención, hasta la causa del choque es desconocida, cada parte soporta sus propias pérdidas; cuando el choque es impuLable a una de las partis, aquella es responsable por todos los daños $\mathrm{y}$, en fin, cuando hay culpa de parte de otro. la responsabilidad de cada una de las partes es proporcional a su culpa.

Este régimen, sin embargo, no será aplicable, en la hora presente, en la repartición de daños ocasionados por las actividades espaciales. Por una parte, los conocimientos técnicos en materia de explotación espacial no son todavía suficientemente precisos, para que, en caso de accidente, se pueda realmente hablar de una culpa como causa primera.

Por otra parte, cuando su causa es conocida, que será la excepzión por largo tiempo todavia, será siempre posible establecer que el accidente pudo ser evitado si una técnica más perfeccionada pudo ser aplicada, o si las condiciones o consecuencias de la actividad espacial fueron apreciadas nás exactamente. Los progresos de la técnica y de sus métodos zno son debidos, a menudo, a acoidentes, en los cuales se ha podido descubrir la causa? Desde luego, se deberá aplicar la regla que cualquiera que participe en la explotación espacial debe soportar los daños resultantes de sus actividades, sin tener la posibilidad de volverse contra los coautores de estos daños.

21. Queda, en fin, la cuestión de saber si es preciso limitar la responsabilidad objetiva impuesta al Estado en que se hace el lanzamiento o al Estado del cual dependen las estaciones terrestres cie control. En cfecio, cuando una ley 
o convención prevén la responsabilidad objetiva, ella fija normalmente un límite al monto de la indemnización pagadera a la víctima. Pero en el caso particular de daños causados por un vehículo espacial parece inoportuno, $y$, en el hecho, imposible prescribir límites de responsabilidad. Los vehículos y sus actividades, más que daños materiales causados a innumerables propietarios, pueaen herir o matal una multitud de personas, destruir regiones enteras y desorganizar la vida de todo un pueblo. No hay, pues, ningún medio de avaluar por anticipado la medida de los daños, susceptibles de calcularse en muchos miliones, hasta a centenares de trillones. En estas condiciones, todo limite de responsabilidad preestablecido será puramente arbitrario. Mas vale, entonces. que la obligación de reparar los daños resultantes de las actividades espaciales sea ilimitada.

\section{IA ACCION POR LOS DAÑOS E INTERESES}

22. EI régimen bosquejado en esta nota no tendrá valor práctico sino a condición de que las víctimas puedan intentar una acción de daños e intereses al Estado en el cual se hace el lanzamiento o al Estado donde están situadas las estaciones de control, responsables del daño.

En caso contrario, la concesión de inciemnización y su monto dependerán de la buena voluntad del poder ejecutivo o legislativo. La situación de las víctimas extranjeras será peor todavía, porque ellas deberán recurrir a la intervención diplomática del Estado donde son originarias.

23. Ningún problema, es cierto, se produce en el caso de que la ley nacional permita desde luego perseguir la responsabilidad del Estado ante los tribunales judiciales o administrativos. Pero este régimen está lejos de ser universal. Será, pues, necesario que la Convención sobre la responsabilidad por daños causados por una actividad espacial comporte el empeño de cada contratante de dar paso ya por medio de la justicia ordinaria o de sus tribunales administrativos, a las acciones de responsabilidad basadas en las disposiciones de la Convención.

Por cierto, este acuerdo tendrá un carácter revolucionario en los países que no conocen todavía la responsabilidad civil del Estado o continúan aplicando el principio de la inmunidad jurisdiccional de los poderes públicos. Pero la reglamentación satisfactoria dé la responsabilidad en materia de actividad espacial es al precio de esta nivelación de costumbres de los Estados modernos.

24. La alternativa consistirá en ra creación de una instancia judicial internacional como aquella que desde ya se ha hecho en el cuadro de ciertas convenciones regionales europeas. Pero un acuerdo sobre tal jurisdicción será más bien difícil de obtener.

Es necesario añadir que las notas que preceden bosquejan un régimen de responsabilidad destinado a servir como base de discusión.

\section{COMENTARIO}

1. Hemos elegido cste artículo, por cuanto presenta novedades jurídicas en sí, señala nuevos caminos a la investigación y pone de manifiesto la situación del derecho aéreo en Chile

2. El artículo en cuestión plantea en forma clara y objetiva un hecho de innegable importancia: los daños producidos por un Estado a otro, por lanzamientos de vehículos espaciales.

La imperfección en el control de los mismos y enormidad de daños que pueden producir, hace no sólo necesario sino que imprescindible, un acuerdo de tipo internacional que asegure a las víctimas de estos lanzamientos una indemnización, pero como lo señala el autor, no podrán regularse, por el monto insospechado a que ptueden elevarse.

Paises, como los nuestros, que por diversas razones, principalmente de tipo económico, están fuera de la carrera por ia conquista del espacio, serán los principales afectados por estos lanzamientos $y$ deben procurar cuanto antes las me- 
didas necesarias para que, en caso de producirse accidentes que signifiquen perjuicios tanto en vidas como materiales, exista una convención internacional anterior que asegure desde ya la responsabilidad del Estado quz efectuó el lanzamiento.

Es preciso entonces, que este acuerdo internacional sirva de freno a las grandes potencias que con mayores recursos técnicos, pretenden conquistar para sí, situaciones que muchas veces, por razones politicas, militares o económicas, van en desmedro de aquelios Estados, como el nuestro, que no tienen ni siquiera los medios para oponerse a ellas.

3. El presente trabajo no es el primero en género. Trata de uno de los tantos problemas que resultan de la conquista del espacio extraterrestre y que ha dado origen al derecho espacial.

La tan discutida frase de sí el derecho debe preceder a los hechos, ya no tiene aplicación en la actualidad, puesto que puede hablarse de un derecho espacial, distinto del derecho aéreo que le sirve de base. Aún aquellos que ven en aquél sólo un nuevo aspecto del derecho aéreo, no pueden dejar de reconocer que existe un conjunto de problemas que no están reglamentados por acuercio internacional alguno y que las reglas existentes no pueden solucionar sus muchas intaro gantes.

Se abre aşi un campo ilimitado para la investigación jurídica.

Las Primeras Jornadas de Derecho Público, celebradas en Santiago por la Escuela de Derecho de la Universidad de Chile, trataron en uno de los foros, el derecho espacial, por primera vez en nuestro pais, llegando a conclusiones que, para satisfacción de aquellos que participaron, están siendo llevadas a la realidad por las Naciones Unidas y diferentes entidades internacionales.

4. Nuestro país, por diversas razones, presenta un panorama frente al derecho aéreo, que hace que éste no tenga en apariencia su verdadero valor respecto del resto de las ramas del derecho.

Nos rige un DFL de 15 de Mayo de 1931, el cual no ha sufrido mayores modificaciones hasta ahora. Podrá creerse entonces que no se ha progresado en el campo de la aviación. No. Chile ha seguido por su conformación y ubicación geográfica un ritmo acelerado dentro de sus posibilidades económicas.

Sin embargo, el legislador permanece indiferente. La técnica ha sobrepasado con creces a la ley.

\title{
IMPORTANCIA DE LA FACILITACION DEL TRANSPORTE AEREO INTERNACIONAL
}

\author{
por PATRICIO BUSQUET IRRIBARRA
}

Ayudante de Derecho Aeleo del Seminario de Derecho Púbheo.

El enorme desarrollo experimentado por el transporte aéreo desde el término de la Segunda Guerra Mundial ha obligado a los Estados a abocarse a la solución de un doble tipo de problemas:

1.-Los problemas técnicos, creados por la continua y rápida perfección de las aeronaves, que en un lapso de diez años han duplicado su velocidad y capacidad de transporte, prediciéndose que la capacidad y velocidad actual de los aviones a reacción será a su vez duplicada en menos de tres años por los apa- 